\title{
Erroneous data generation for Grammatical Error Correction
}

\author{
Shuyao Xu Jiehao Zhang Jin Chen Long Qin \\ Singsound Inc. \\ \{xushuy, zhangjiehao, chenjin, qinlong\}@singsound. com
}

\begin{abstract}
It has been demonstrated that the utilization of a monolingual corpus in neural Grammatical Error Correction (GEC) systems can significantly improve the system performance. The previous state-of-theart neural GEC system is an ensemble of four Transformer models pretrained on a large amount of Wikipedia Edits. The Singsound GEC system follows a similar approach but is equipped with a sophisticated erroneous data generating component. Our system achieved an $F_{0.5}$ of 66.61 in the BEA 2019 Shared Task: Grammatical Error Correction. With our novel erroneous data generating component, the Singsound neural GEC system yielded an $\mathrm{M}^{2}$ of 63.2 on the CoNLL-2014 benchmark $(8.4 \%$ relative improvement over the previous state-of-the-art system).
\end{abstract}

\section{Introduction}

The most effective approaches to Grammatical Error Correction (GEC) task are machine translation based methods. Both Statistical Machine Translation (SMT) approaches and Neural Machine Translation (NMT) methods have achieved promising results in the GEC task.

Pretraining a decoder as a language model is an effective method to improve the performance of neural GEC systems (Junczys-Dowmunt et al., 2018). As an extension of this work, Lichtarge et al. (2018) showed pretraining on 4 billion tokens of Wikipedia edits to be beneficial for the GEC task.

In this work, we investigate a similar approach by systematically generating parallel data for pretraining. As shown in Table 1, in addition to spelling errors (price $\rightarrow$ puice), transposition errors (independent voters $\rightarrow$ voters independent) and concatenation errors (the man $\rightarrow$ theman), our

\begin{tabular}{l|l}
\hline Origin & $\begin{array}{l}\text { the primary is open to independent } \\
\text { voters . } \\
\text { the primary is opens to voters } \\
\text { independhent } .\end{array}$ \\
\hline Origin & $\begin{array}{l}\text { the price of alcohol is ramped up } \\
\text { at every budget . } \\
\text { the puice of alchool is ramping up } \\
\text { at every budget . }\end{array}$ \\
Generated \\
Origin & $\begin{array}{l}\text { they say the police shot and killed } \\
\text { the man after he had fired at them } \\
\text { they say the polices shot and killed } \\
\text { theman after he had firing at them . }\end{array}$ \\
Generated
\end{tabular}

Table 1: Examples of generated data.

method also introduces errors such as ramped $\rightarrow$ ramping. Our approach obtained competitive results compared to the top systems in the BEA 2019 GEC Shared Task. Both our single model and ensemble models have exceeded the previous stateof-the-art systems on the CoNLL-2014 (Ng et al., 2014) benchmark and our system reaches humanlevel performance on the JFLEG (Napoles et al., 2017) benchmark.

\section{Related Work}

Chollampatt and $\mathrm{Ng}$ (2018) used a convolutional sequence-to-sequence (seq2seq) model (Gehring et al., 2017) with a large language model for rescoring. Their model was the first NMT based GEC system that exceeded the strong SMT baseline system (Junczys-Dowmunt and Grundkiewicz, 2016) which combined a Phrase-based Machine Translation (PBMT) with a large language model. Then a hybrid PBMT-NMT system (Grundkiewicz and Junczys-Dowmunt, 2018) appeared to reach the new state-of-the-art on the CoNLL-2014 benchmark. Later, various pure neu- 


\begin{tabular}{|l|l|l|l|}
\hline Corpus & Sentences & Tokens & Anno. \\
\hline WMT11 & $115 \mathrm{M}$ & $2362 \mathrm{M}$ & No \\
1B words & $30 \mathrm{M}$ & $769 \mathrm{M}$ & No \\
Lang-8 & $1037 \mathrm{~K}$ & $12 \mathrm{M}$ & Yes \\
NUCLE & $57 \mathrm{~K}$ & $1.2 \mathrm{M}$ & Yes \\
FCE & $28 \mathrm{~K}$ & $455 \mathrm{~K}$ & Yes \\
ABCN & $34 \mathrm{~K}$ & $628 \mathrm{~K}$ & Yes \\
\hline
\end{tabular}

Table 2: Statistics for training data sets.

\begin{tabular}{|l|l|l|}
\hline Corpus & Sentences & Scorer \\
\hline ABCN dev & 4384 & ERRANT \\
ABCN test & 4477 & ERRANT \\
JFLEG test & 747 & GLEU \\
CoNLL-2014 test & 1312 & $\mathrm{M}^{2}$ Scorer \\
\hline
\end{tabular}

Table 3: Statistics for test and development data.

ral systems (Ge et al., 2018; Junczys-Dowmunt et al., 2018; Lichtarge et al., 2018) reported stateof-the-art results successively. Ge et al. presented the fluency boosting method which was demonstrated to be effective to improve performance of GEC seq2seq models. The system proposed by Junczys-Dowmunt et al. (2018) is an ensemble of Transformer models (Vaswani et al., 2017); they pretrained the decoder of transformer as a language model on a large monolingual corpus. To our best knowledge, the current state-of-the-art GEC system on both the CoNLL-2014 benchmark and the JFLEG benchmark is the system presented by Lichtarge et al. (2018), which is an ensemble of four Transformer models pretrained on Wikipedia revisions and then fine-tuned on Lang-8 (Mizumoto et al., 2011).

\section{Data}

We list the training data in Table 2. The text data used to generate parallel corpus automatically was the One Billion Words Benchmark dataset (1B words) (Chelba et al., 2013) and the WMT11 monolingual corpus (WMT11) which can be obtained from WMT11 Website $^{1}$. Our fine-tuning data is Lang-8 (Mizumoto et al., 2011; Tajiri et al., 2012), NUS Corpus of Learner English (NUCLE) (Dahlmeier et al., 2013), FCE (Yannakoudakis et al., 2011), the Cambridge English Write \& Improve (W\&I) corpus and the LOCNESS corpus (ABCN) (Granger, 1998; Bryant et al., 2019) .

\footnotetext{
${ }^{1}$ http://statmt.org/wmt11/ training-monolingual.tgz
}

\begin{tabular}{|c|c|c|c|c|c|}
\hline Length & Err. & Prob. & Length & Err. & Prob. \\
\hline \multirow{2}{*}{1,3} & 0 & 0.50 & \multirow{3}{*}{6,9} & 2 & 0.30 \\
\hline & 1 & 0.50 & & 3 & 0.45 \\
\hline \multirow{2}{*}{3,6} & 1 & 0.50 & & 4 & 0.25 \\
\hline & 2 & 0.50 & \multirow{5}{*}{16,20} & 3 & 0.10 \\
\hline \multirow{4}{*}{9,16} & 3 & 0.15 & & 4 & 0.15 \\
\hline & 4 & 0.25 & & 5 & 0.15 \\
\hline & 5 & 0.30 & & 6 & 0.30 \\
\hline & 6 & 0.30 & & 7 & 0.30 \\
\hline \multirow{5}{*}{20,30} & 4 & 0.10 & \multirow{5}{*}{$30, \infty$} & 5 & 0.10 \\
\hline & 5 & 0.15 & & 6 & 0.15 \\
\hline & 6 & 0.15 & & 7 & 0.15 \\
\hline & 7 & 0.30 & & 8 & 0.30 \\
\hline & 8 & 0.30 & & 9 & 0.30 \\
\hline
\end{tabular}

Table 4: Probability distribution of sentence errors.

Table 3 shows the development and test data sets in our experiments. We choose the $\mathrm{ABCN}$ dev set as our development set and the ABCN test, the CoNLL-2014 test, the JFLEG as our benchmark. For these benchmarks, we report precision $(P)$, recall $(R)$ and $F_{0.5}$ with ERRANT (Bryant et al., 2017) on the ABCN test, GLEU (Sakaguchi et al., 2016) on the JFLEG test set (Napoles et al., 2017). To compare with previous state-of-the-art systems, we provide results of MaxMatch $\left(\mathrm{M}^{2}\right)$ Scorer (Dahlmeier and Ng, 2012) on the CoNLL2014 test set.

\section{Erroneous Data Generation}

In this section, we describe our error generating method. For each sentence, we assign a probability distribution (as shown in Table 4) to determine the number of errors according to the sentence length. The parameters in Table 4 are determined empirically, as well as the parameters in Table 5, Table 6 and Table 7. Because of the time limitation of the GEC competition, we did not optimize these parameters.

After the number of errors (E) in a sentence has been determined, we randomly select $\mathrm{E}$ tokens from all the tokens of the sentence with equal probability to be errors. And for each error, we apply a random variable (Table 5) to determine which error type it should be.

We introduce five error types:

- Concatenation: combine two consecutive tokens, e.g., hello world $\rightarrow$ helloworld.

- Misspelling: introduce spelling errors into 


\begin{tabular}{|l|l|}
\hline Type & Prob. \\
\hline Concatenation & 0.12 \\
Misspell & 0.45 \\
Substitution & 0.40 \\
Deletion & 0.00 \\
Transposition & 0.03 \\
\hline
\end{tabular}

Table 5: Error types.

\begin{tabular}{|lll|}
\hline Tok. length & Err. & Prob. \\
\hline$[1,3)$ & 0 & 1.00 \\
\hline$[3,5)$ & 1 & 1.00 \\
\hline$[5,10)$ & 1 & 0.80 \\
& 2 & 0.20 \\
\hline$[10, \infty)$ & 1 & 0.75 \\
& 2 & 0.15 \\
& 3 & 0.10 \\
\hline
\end{tabular}

Table 6: Number of misspells in a token.

\begin{tabular}{|l|l|}
\hline Type & Prob. \\
\hline Deletion & 0.30 \\
Insertion & 0.15 \\
Transposition & 0.25 \\
Replacement & 0.30 \\
\hline
\end{tabular}

Table 7: Mispell types.

words, e.g., computer $\rightarrow$ camputer.

- Substitution: we introduce seven different types of substitutions.

- Deletion: delete the token.

- Transposition: the token exchange position with a consecutive token.

\subsection{Misspelling}

To generate misspellings, we introduce a random variable to determine how many errors in the token according to the token length (parameters are shown in Table 6.), and we randomly insert errors into the token.

For each spelling error, we apply another random variable to determine which error type should be. We introduce four spelling error types (Table 7 lists the parameters.).

- Deletion: delete the character.

- Insertion: insert a random English letter into the current position.
- Transposition: exchange position with the consecutive character.

- Replacement: replace the current character with a random English character.

We only introduce spelling errors into words belonging to a vocabulary list of $32 \mathrm{k}$ ordinary words ${ }^{2}$ which does not include numerals (e.g., 2019), tokens that contain digits (e.g., Lang8), URLs or non-word symbols (e.g., $\geq \nabla \leq)$.

\subsection{Substitution}

We introduce seven types of substitutions according to token and its part-of-speech (POS).

- Substitution between Prepositions. E.g., in, on, at, through, for, with.

- Substitution between Articles. E.g., a, an, the.

- Substitution between Pronouns (Singular). E.g., he, she, his, him, her, hers.

- Substitution between Pronouns (Plural). E.g., their, them, they, theirs.

- Substitution between Wh words. E.g., which, where, what, how, when, who, whose, whom.

- Substitution between Modal verbs. E.g., will, shall, can, may, would, could, might.

- Substitution in a Word Tree (see 4.3 for details).

\subsection{Word Tree}

We want to make substitutions such as going $\rightarrow$ gone, useful $\rightarrow$ usable, administration $\rightarrow$ administrative. To make such substitution possible, we introduce the Word Tree.

A Word Tree represents a group of words that share the same stem but have different suffixes. Figure 1 shows an example of Word Tree of "use". A node denotes a word (e.g., usable) and corresponding Extended part-of-speech (EPOS) (e.g., VBP_JJ_BLE) (see 4.4 for details.), and an edge indicates the root from which the word is derived (e.g., "usable" is derived from "use").

With EPOS, we can easily set rules or assign probability distributions to determine which substitutions are more likely to happen, (e.g., singular $\leftrightarrow$ plural, $\mathrm{VBD} \leftrightarrow \mathrm{VBZ} \leftrightarrow \mathrm{VBP} \leftrightarrow \mathrm{VBN} \leftrightarrow$

\footnotetext{
${ }^{2}$ We manually created this vocabulary for building the Word Tree (see 4.3 for details).
} 


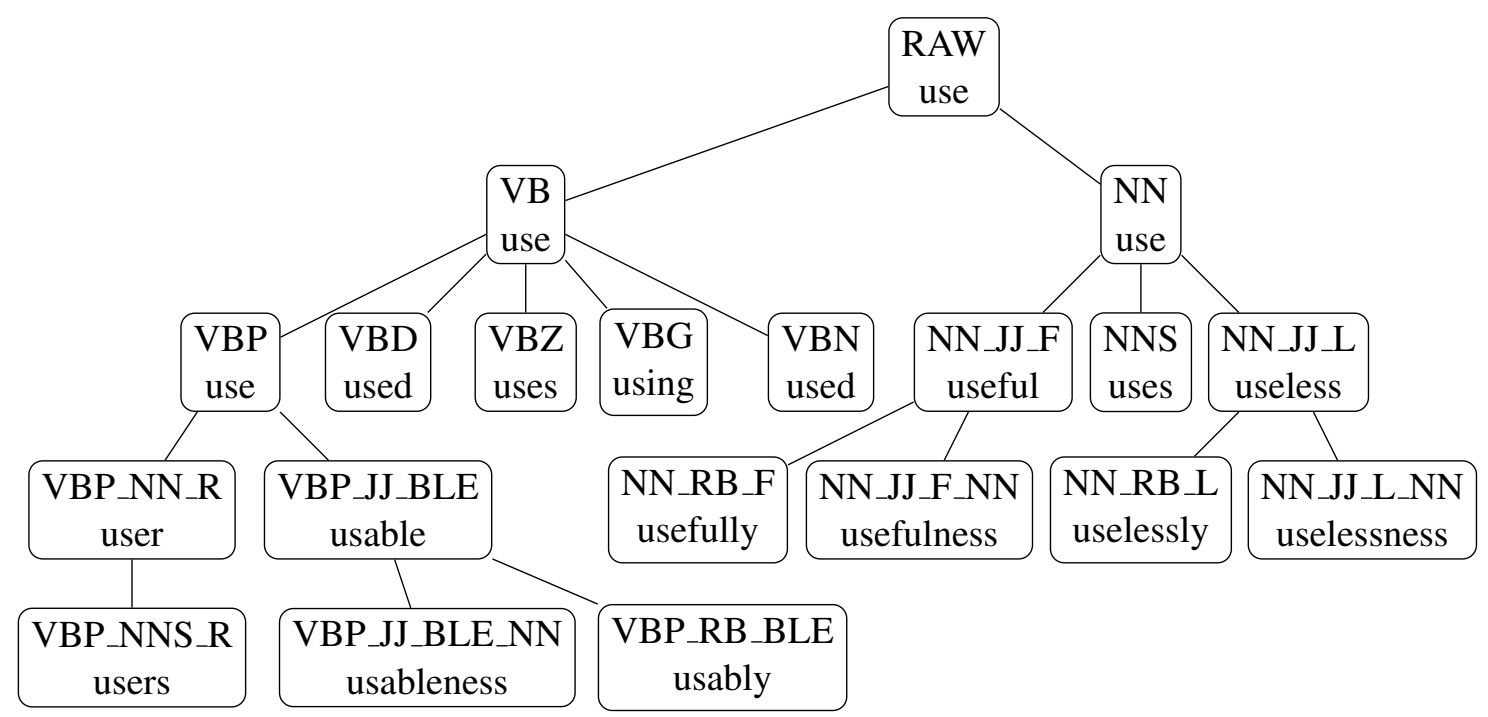

Figure 1: Word Tree: use

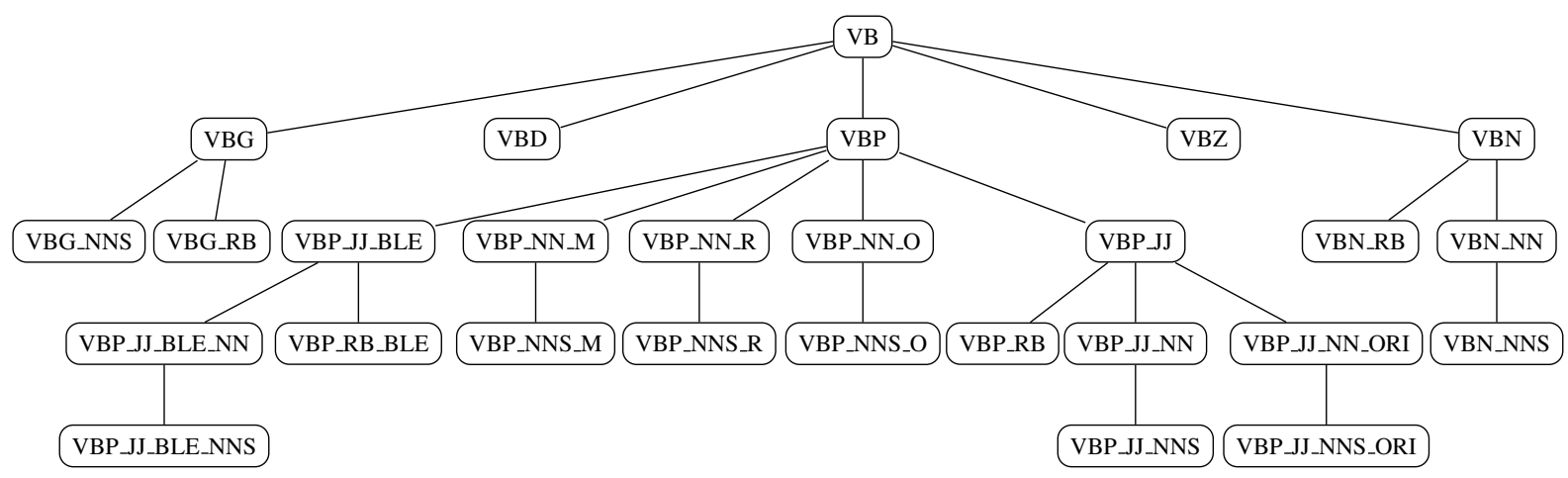

Figure 2: Verb branch of the EPOS Tree

VBG, adjective $\leftrightarrow$ adverb), and which substitutions are less likely to happen (e.g., happiest JJS $\leftrightarrow$ happiness JJ_NN). In our experiments, due to the time limitation of the competition, we simply assigned a uniform distribution to all existing words in a Word Tree, excluding substitutions that were definitely unlikely to occur such as substitutions between the words in an NN_JJ_F branch (e.g., careful) and the words in an NN_JJ_L branch (e.g., carelessness).

\subsection{Extended part-of-speech}

A Word Tree can contain multiple words of the same POS. As shown in the example in Figure 1, use, user and usefulness can all be nouns. Therefore, in order to identify the different roles for words in a Word Tree, we propose EPOS, derived from part-of-speech (POS) and the surface form of the word.

POS explains how a word is used (mostly syn- tactically) in a sentence. Compared to POS, EPOS also reflects some semantic role of a word in a sentence.

We define EPOS in Table 11 in the Appendix. We used NLTK (Bird, 2006) as our POS tagger, and use NLTK-style tags in this paper.

We briefly describe our method of creating word trees.

a. Extract the vocabulary from a text corpus which is tagged with NLTK POS tagger.

b. Create three tables for Noun, Verb, and Adjective respectively. The Noun table contains two columns: singular and plural; the Verb table has six columns: original verb form (VB), non-third person present (VBP), third person present (VBZ), past tense (VBD), past participle (VBN) and present participle (VBG); the Adjective table has four columns: adjective, adverb, comparative degree and superlative de- 


\begin{tabular}{lllll}
\hline$\#$ & System & $P$ & $R$ & $F_{0.5}$ \\
\hline 1 & UEDIN-MS & 72.28 & 60.12 & 69.47 \\
& $\ldots$ & & & \\
4 & CAMB-CLED & 70.49 & 55.07 & 66.75 \\
5 & Singsound & 70.17 & 55.39 & 66.61 \\
\hline
\end{tabular}

Table 8: Results of BEA 2019 GEC competition.

\begin{tabular}{c|l|lll}
\hline & dev & \multicolumn{3}{|l}{ test } \\
& $F_{0.5}$ & $P$ & $R$ & $F_{0.5}$ \\
\hline single & 52.29 & 66.06 & 56.68 & 63.94 \\
w/o pretrain & 44.60 & 50.59 & 43.60 & 49.02 \\
\hline 4 ensemble & 55.37 & 70.14 & 57.57 & 67.21 \\
w/o pretrain & 47.01 & 56.05 & 44.33 & 53.24 \\
\hline
\end{tabular}

Table 9: Results of ABCN set. "w/o pretrain" refers to models without pretraining.

gree. Then we fill words into corresponding entries according to their POS tags. Words that cannot be filled in any of the above tables are filled into a list.

c. Manually check and correct all entries of the three tables, and fill missing entries as well.

d. Define EPOS as listed in Table 11 in the Appendix according to suffix transforming rules.

e. Extract a RAW list from the vocabulary according to the suffix transforming rules.

f. Create an EPOS tree structure for each token in the RAW list, and then fill each word from the vocabulary into the corresponding entry of the corresponding EPOS tree (The full structure of the EPOS Tree is described in Table 12 in the Appendix, and Figure 2 shows the Verb branch); then prune empty entries in the trees.

g. Manually check every entry of every word tree, and fix all incorrect entries.

h. Update the defined EPOS (final version in Table 11) and the EPOS tree (Table 12); recreate word trees.

i. Repeat step g and h until satisfied.

\section{Experiments}

In our experiments, we generated a corpus of 3 billion tokens, of which about $24 \%$ were errors.
Following Lichtarge et al. (2018), we also use Transformer as our encoder-decoder model, using Tensor2Tensor open source implementation ${ }^{3}$.

The models are trained on words, and rare words are segmented into sub-words with the byte pair encoding (BPE) (Sennrich et al., 2015). We use 6 layers for both encoder and decoder, and 4 attention heads. The embedding size and hidden size are 1024, and the filter size for all positionwise feed forward network is 4096 . We set dropout rate to 0.3 , and source word dropout is set to 0.2 as a noising technique. Following JunczysDowmunt et al. (2018), source, target and output embeddings are tied in our models.

Following Lichtarge et al. (2018), we first trained our model on an artificially generated parallel corpus with a batch size of approximately 3072 tokens. Then we set the batch size to 2048 tokens and fine-tuned on human annotated data for 20 epochs, and we averaged the 5 best checkpoints. Finally, the averaged model was fine-tuned on the ABCN and FCE training data for 1000 steps as domain adaptation (Junczys-Dowmunt et al., 2018).

There are about $50 \%$ sentence pairs without any correction in the Lang- 8 dataset, and we noticed that training with too many error-free sentence pairs had a negative effect. Therefore, we filtered out these error-free sentence pairs in the Lang8 dataset. Since the NUCLE, FCE and ABCN datasets are much smaller than the Lang-8 set, we did not filter out the error-free sentence pairs in these datasets.

We used beam search for decoding with a beam size of 4 at evaluation time. For the ensemble, we averaged logits from 4 Transformer models with identical hyper-parameters at each decoding step. Following (Grundkiewicz and JunczysDowmunt, 2018; Junczys-Dowmunt et al., 2018; Lichtarge et al., 2018), we preprocessed the JFLEG dataset with spell-checking. We did not apply spell-checking to the ABCN and CoNLL-2014 datasets.

\section{Results and Discussion}

The results of the Singsound System in the GEC competition (Table 8) were obtained by an ensemble of four models. Because of the time limitation, we only trained two independent models

\footnotetext{
${ }^{3}$ https://github.com/ tensorflow/tensor2tensor
} 


\begin{tabular}{l|c|ccc|ccc|c}
\hline \multirow{2}{*}{ Model } & \multicolumn{3}{|c|}{ CoNLL-2014 } & CoNLL-10 (SvH) & JFLEG \\
& Word\&Char SMT-GEC & 62.7 & 33.0 & 53.1 & & & 68.3 & 56.8 \\
\hline$(1)$ & MLConv (4 ensemble) & 65.5 & 33.1 & 54.8 & & & & 57.5 \\
\hline$(2)$ & Transformer (single) & & & 53.0 & & & & 57.9 \\
\hline \multirow{3}{*}{$(3)$} & Transformer (4 ensemble) & 63.0 & 38.9 & 56.1 & & & & 58.5 \\
& Transformer (4 ensemble) + LM & 61.9 & 40.2 & 55.8 & & & & 59.9 \\
\hline \multirow{2}{*}{$(4)$} & Hybrid PBMT+NMT+LM & 66.8 & 34.5 & 56.3 & 83.2 & 47.0 & 72.0 & 61.5 \\
\hline \multirow{2}{*}{$(5)$} & Transformer (single) & 62.2 & 37.8 & 54.9 & & & & 59.3 \\
& Transformer (4 ensemble) & 67.5 & 37.8 & 58.3 & & & & 62.4 \\
\hline \multirow{2}{*}{ Singsound } & Transformer (single) & 68.3 & $\mathbf{4 2 . 5}$ & 60.9 & 83.5 & 55.2 & 75.7 & 60.8 \\
& Transformer (4 ensemble) & $\mathbf{7 3 . 0}$ & 41.1 & $\mathbf{6 3 . 2}$ & $\mathbf{8 6 . 0}$ & 53.8 & $\mathbf{7 6 . 8}$ & $\mathbf{6 2 . 6}$ \\
\hline & Human avg. & & & 73.5 & $\mathbf{6 9 . 6}$ & 72.6 & 62.4 \\
\hline
\end{tabular}

Table 10: Comparison with top performing systems on CoNLL and JFLEG datasets. (1): Chollampatt and Ng (2017) (2): Chollampatt and Ng (2018); (3): Junczys-Dowmunt et al. (2018); (4): Grundkiewicz and JunczysDowmunt (2018); (5): Lichtarge et al.(2018).

from scratch. The other two were based on existing trained models. Concretely, after we got a model trained from scratch, we kept training it on the generated corpus for 0.2 epoch; then fine-tuned the model on the annotated data and $\mathrm{ABCN}$ and FCE training sets as before.

We provide the performance of our single model and the ensemble of 4 independently trained models ${ }^{4}$ on the ABCN dev and test datasets in Table 9. As the results shown in Table 9, models pretrained on the generated corpus significantly outperform the models without pretraining.

To compare with previous state-of-the-art GEC systems, we evaluated our systems on the CoNLL2014 and JFLEG datasets. As the results shown in Table 10, our single model exceeded previous state-of-the-art systems on the CoNLL-2014 dataset. Our ensemble models achieved $8.4 \%$ relative improvement over the latest state-of-the-art results on the CoNLL-2014 benchmark.

We also report the results on the CoNLL-2014 10 annotation dataset (denoted as CoNLL-10) (Bryant and $\mathrm{Ng}, 2015$ ) which is an extension of the CoNLL-2014 test set with 10 annotators. The human-level scores are calculated by averaging the scores for each annotator with regard to the remaining annotators. Following Chollampatt and $\mathrm{Ng}$ (2017), scores on CoNLL-10 (SvH) are calcu-

\footnotetext{
${ }^{4}$ The four models are trained on the same data with the same hyper-parameter set.
}

lated by removing one set of human annotations at a time and evaluating the system against the remaining sets. Our models reach human-level performance on both CoNLL-10 and JFLEG benchmarks.

\section{Conclusion}

In this work, we present a novel erroneous data generating method for training English GEC models. Our experiments show that Transformer models pretrained on generated corpus significantly outperform the previous GEC systems that are also based on Transformer. We also present a novel tool: the Word Tree, which represents a group of words that share the same stem but have different suffixes; and we show that one possible application of the Word Tree is generating erroneous text for training GEC models.

\section{Acknowledgments}

We thank Xiaoxue Fan, Miao Xue and Yueming Gao for their help in checking the three tables required for creating the Word Trees.

\section{References}

Steven Bird. 2006. NLTK: The natural language toolkit. meeting of the Association for Computational Linguistics, pages 69-72. 
Christopher Bryant, Mariano Felice, Øistein E. Andersen, and Ted Briscoe. 2019. The BEA-2019 Shared Task on Grammatical Error Correction. In Proceedings of the 14th Workshop on Innovative Use of NLP for Building Educational Applications. Association for Computational Linguistics.

Christopher Bryant, Mariano Felice, and Edward John Briscoe. 2017. Automatic annotation and evaluation of error types for grammatical error correction. Association for Computational Linguistics.

Christopher Bryant and Hwee Tou Ng. 2015. How far are we from fully automatic high quality grammatical error correction. 1:697-707.

Ciprian Chelba, Tomas Mikolov, Mike Schuster, Qi Ge, Thorsten Brants, Phillipp Koehn, and Tony Robinson. 2013. One billion word benchmark for measuring progress in statistical language modeling. Computer Science.

Shamil Chollampatt and Hwee Tou Ng. 2017. Connecting the dots: Towards human-level grammatical error correction. pages 327-333.

Shamil Chollampatt and Hwee Tou Ng. 2018. A multilayer convolutional encoder-decoder neural network for grammatical error correction. In Thirty-Second AAAI Conference on Artificial Intelligence.

Daniel Dahlmeier and Hwee Tou Ng. 2012. Better evaluation for grammatical error correction. In Proceedings of the 2012 Conference of the North American Chapter of the Association for Computational Linguistics: Human Language Technologies, pages 568-572. Association for Computational Linguistics.

Daniel Dahlmeier, Hwee Tou Ng, and Siew Mei Wu. 2013. Building a large annotated corpus of learner English: The NUS corpus of learner English. In Proceedings of the eighth workshop on innovative use of NLP for building educational applications, pages 22-31.

Tao Ge, Furu Wei, and Ming Zhou. 2018. Fluency boost learning and inference for neural grammatical error correction. In Proceedings of the 56th Annual Meeting of the Association for Computational Linguistics (Volume 1: Long Papers), pages 10551065 .

Jonas Gehring, Michael Auli, David Grangier, Denis Yarats, and Yann N Dauphin. 2017. Convolutional sequence to sequence learning. In Proceedings of the 34th International Conference on Machine Learning-Volume 70, pages 1243-1252. JMLR. org.

Sylviane Granger. 1998. The computer learner corpus: a versatile new source of data for SLA research. na.

Roman Grundkiewicz and Marcin Junczys-Dowmunt. 2018. Near human-level performance in grammatical error correction with hybrid machine translation. arXiv preprint arXiv:1804.05945.
Marcin Junczys-Dowmunt and Roman Grundkiewicz. 2016. Phrase-based machine translation is state-ofthe-art for automatic grammatical error correction. arXiv preprint arXiv:1605.06353.

Marcin Junczys-Dowmunt, Roman Grundkiewicz, Shubha Guha, and Kenneth Heafield. 2018. Approaching neural grammatical error correction as a low-resource machine translation task. arXiv preprint arXiv:1804.05940.

Jared Lichtarge, Christopher Alberti, Shankar Kumar, Noam Shazeer, and Niki Parmar. 2018. Weakly supervised grammatical error correction using iterative decoding. arXiv preprint arXiv:1811.01710.

Tomoya Mizumoto, Mamoru Komachi, Masaaki Nagata, and Yuji Matsumoto. 2011. Mining revision log of language learning SNS for automated Japanese error correction of second language learners. In Proceedings of 5th International Joint Conference on Natural Language Processing, pages $147-155$

Courtney Napoles, Keisuke Sakaguchi, and Joel Tetreault. 2017. JFLEG: A fluency corpus and benchmark for grammatical error correction. arXiv preprint arXiv:1702.04066.

Hwee Tou Ng, Siew Mei Wu, Ted Briscoe, Christian Hadiwinoto, Raymond Hendy Susanto, and Christopher Bryant. 2014. The conll-2014 shared task on grammatical error correction. In Proceedings of the Eighteenth Conference on Computational Natural Language Learning: Shared Task, pages 1-14.

Keisuke Sakaguchi, Courtney Napoles, Matt Post, and Joel R Tetreault. 2016. Reassessing the goals of grammatical error correction: Fluency instead of grammaticality. Transactions of the Association for Computational Linguistics, 4(1):169-182.

Rico Sennrich, Barry Haddow, and Alexandra Birch. 2015. Neural machine translation of rare words with subword units. arXiv preprint arXiv:1508.07909.

Toshikazu Tajiri, Mamoru Komachi, and Yuji Matsumoto. 2012. Tense and aspect error correction for esl learners using global context. pages 198-202.

Ashish Vaswani, Noam Shazeer, Niki Parmar, Jakob Uszkoreit, Llion Jones, Aidan N Gomez, Łukasz Kaiser, and Illia Polosukhin. 2017. Attention is all you need. In Advances in neural information processing systems, pages 5998-6008.

Helen Yannakoudakis, Ted Briscoe, and Ben Medlock. 2011. A new dataset and method for automatically grading ESOL texts. In Proceedings of the 49th Annual Meeting of the Association for Computational Linguistics: Human Language Technologies-Volume 1, pages 180-189. Association for Computational Linguistics. 


\section{A Appendix}

\begin{tabular}{|c|c|c|c|}
\hline EPOS & POS & Annotation & Examples \\
\hline RAW & & Root of word trees, original form & use \\
\hline $\mathrm{NN}$ & NN & Noun & use \\
\hline NNS & NNS & Plural form of Noun & uses \\
\hline NN_JJ_F & JJ & $\mathrm{NN}+$ ful $^{5}$ & useful \\
\hline NN_JJ_F_NN & $\mathrm{NN}$ & NN_JJ_F + ness & usefulness \\
\hline NN_JJ_F_NNS & NNS & Plural form of NN_JJ_F_NN & \\
\hline NN_JJ_F_NN_ORI & $\mathrm{NN}$ & Adjective used as Noun & dreadful \\
\hline NN_JJ_F_NNS_ORI & NNS & Plural form of NN_JJ_F_NN_ORI & dreadfuls \\
\hline NN_RB_F & $\mathrm{RB}$ & Adverb form of NN_JJ_F & usefully \\
\hline NN_JJ_L & $\mathrm{JJ}$ & $\mathrm{NN}+$ less & useless \\
\hline NN_JJ_L_NN & $\mathrm{NN}$ & NN_JJ_L + ness & uselessness \\
\hline NN_JJ_L_NNS & NNS & Plural form of $\mathrm{NN} \_J J \_L \_N N$ & \\
\hline NN_JJ_L_NN_ORI & NN & Adjective used as Noun & wireless \\
\hline NN_JJ_L_NNS_ORI & NNS & Plural form of NN_JJ_L_NN_ORI & wirelesses \\
\hline NN_RB_L & $\mathrm{RB}$ & Adverb form of NN_JJ_L & uselessly \\
\hline NN_JJ_OUS & $\mathrm{JJ}$ & $\mathrm{NN}+$ ous & dangerous \\
\hline NN_JJ_OUS_NN & NN & NN_JJ_OUS + ness & dangerousness \\
\hline NN_JJ_OUS_NNS & NNS & Plural form of NN_JJ_OUS_NN & \\
\hline NN_RB_OUS & $\mathrm{RB}$ & Adverb form of NN_JJ_OUS & dangerously \\
\hline NN_JJ_AL & $\mathrm{JJ}$ & $\mathrm{NN}+\mathrm{al}$ & rational \\
\hline NN_JJ_AL_NN & $\mathrm{NN}$ & NN_JJ_AL + ness & rationalness \\
\hline NN_JJ_AL_NNS & NNS & Plural form of $\mathrm{NN} \_J J \_A L \_N N$ & \\
\hline NN_RB_AL & $\mathrm{RB}$ & Adverb form of NN_JJ_AL & rationally \\
\hline NN_JJ_Y & $\mathrm{JJ}$ & $\mathrm{NN}+\mathrm{y}$ & lucky \\
\hline NN_JJR_Y & JJR & Comparative degree of NN_JJ_Y & luckier \\
\hline NN_JJS_Y & JJS & Superlative degree of NN_JJ_Y & luckiest \\
\hline NN_JJ_Y_NN & NN & NN_JJ_Y + ness & luckiness \\
\hline NN_JJ_Y_NNS & NNS & Plural form of NN_JJ_Y_NN & \\
\hline NN_JJ_Y_NN_ORI & $\mathrm{NN}$ & Adjective used as Noun & safety \\
\hline NN_JJ_Y_NNS_ORI & NNS & Plural form of NN_JJ_Y_NN_ORI & safeties \\
\hline NN_RB_Y & $\mathrm{RB}$ & Adverb form of ${\mathrm{NN} \_J J_{-} \mathrm{Y}}$ & luckily \\
\hline NN_JJ_D & $\mathrm{JJ}$ & $\mathrm{NN}+\mathrm{ed}$ & warmhearted \\
\hline NN_JJ_D_NN & $\mathrm{NN}$ & NN_JJ_D + ness & warmheartedness \\
\hline NN_JJ_D_NNS & NNS & Plural form of NN_JJ_D_NN & \\
\hline NN_RB_D & $\mathrm{RB}$ & Adverb form of $\mathrm{NN} \_J J \_\mathrm{D}$ & warmheartedly \\
\hline VB & VB & Original form of verbs & go \\
\hline VBD & VBD & Past tense & went \\
\hline VBZ & VBZ & Present third person singular & goes \\
\hline VBN & VBN & Past participle & gone \\
\hline VBN_NN & NN & VBN + ness & limitedness \\
\hline VBN_NNS & NNS & Plural form of VBN_NN & \\
\hline VBN_NNS_ORI & NNS & Plural form of VBN when VBN used as Noun & shots, thoughts \\
\hline VBN_RB & $\mathrm{RB}$ & Adverb form of VBN & excitedly \\
\hline VBG & VBG & Present participle & baking \\
\hline VBG_NNS_ORI & NNS & Plural form of VBG when VBG used as Noun & bakings \\
\hline VBG_RB & RB & Adverb form of VBG & excitingly \\
\hline VBP & VBP & non-third person present & go \\
\hline
\end{tabular}




\begin{tabular}{|c|c|c|c|}
\hline VBP_NN_O & $\mathrm{NN}$ & $\mathrm{VBP}+$ ion & connection \\
\hline VBP_NNS_O & NNS & Plural form of VBP_NN_O & connections \\
\hline VBP_NN_R & NN & $\mathrm{VBP}+\mathrm{er} /$ or $/ \mathrm{ar}$ & dancer, editor \\
\hline VBP_NNS_R & NNS & Plural form of VBP_NN_R & dancers, editors \\
\hline VBP_JJ_BLE & $\mathrm{JJ}$ & VBP + able / ible & usable \\
\hline VBP_JJ_BLE_NN & NN & VBP_JJ_BLE + ness & usableness \\
\hline VBP_JJ_BLE_NNS & NNS & Plural form of VBP_JJ_BLE_NN & \\
\hline VBP_RB_BLE & RB & Adverb form of VBP_JJ_BLE & usably \\
\hline VBP_JJ & $\mathrm{JJ}$ & VBP + ive & active \\
\hline VBP_RB & RB & Adverb form of VBP_JJ & actively \\
\hline VBP_JJ_NN & $\mathrm{NN}$ & VBP_JJ + ness & attractiveness \\
\hline VBP_JJ_NNS & NNS & Plural form of VBP_JJ_NN & \\
\hline VBP_JJ_NN_ORI & $\mathrm{NN}$ & VBP_JJ used as Noun & representative \\
\hline VBP_JJ_NNS_ORI & NNS & Plural form of VBP_JJ_NN_ORI & representatives \\
\hline VBP_NN_M & $\mathrm{NN}$ & $\mathrm{VBP}+$ ment & movement \\
\hline VBP_NNS_M & NNS & Plural form of VBP_NN_M & movements \\
\hline $\mathrm{JJ}$ & $\mathrm{JJ}$ & Adjectivel & happy \\
\hline JJS & JJS & Superlative degree of Adjective & happiest \\
\hline JJR & JJR & Comparative degree of Adjective & happier \\
\hline $\mathrm{JJ} \_\mathrm{NN}$ & NN & $\mathrm{JJ}+$ ness & happiness \\
\hline JJ_NNS & NNS & Plural form of JJ_NN & happinesses \\
\hline $\mathrm{RB}$ & RB & Adverb & happily \\
\hline RBR & RBR & Comparative degree of Adverb & harder \\
\hline RBS & RBS & Superlative degree of Adverb & hardest \\
\hline $\mathrm{CD}$ & $\mathrm{CD}$ & Cardinal digits & one \\
\hline CD_JJ & $\mathrm{JJ}$ & Adjective form of CD & first \\
\hline CD_RB & RB & Adverb form of numbers & firstly \\
\hline CD_JJ_NN_ORI & $\mathrm{NN}$ & Adjective used as Noun & first \\
\hline CD_JJ_NNS_ORI & NNS & Plural form of CD_JJ_NN_ORI & firsts \\
\hline CD_RB_ORI & $\mathrm{RB}$ & Adverbs that are same as CD_JJ & first \\
\hline CD_NNS & NNS & Plural form of CD & ones \\
\hline DT & DT & Determiner & the \\
\hline WRB & WRB & Wh-adverb & how, where \\
\hline PRP & PRP & Personal pronoun & I, you, they \\
\hline IN & IN & Preposition or subordinating conjunction & in, from, after \\
\hline $\mathrm{CC}$ & $\mathrm{CC}$ & Coordinating conjunction & and \\
\hline MD & MD & Modal verb & can \\
\hline OFS & & Any POS out of the POS column & \\
\hline
\end{tabular}

Table 11: EPOS table.

\footnotetext{
${ }^{5}$ By abuse notation, "+" denotes "with some suffix".
} 


\begin{tabular}{|l|l|}
\hline Parent & Children \\
\hline RAW & NN,JJ, VB, IN, OFS, CC, MD, DT, PRP, CD, WDT, WP, WRB \\
\hline NN & NNS, NN_JJ_F, NN_JJ_L, NN_JJ_Y, NN_JJ_D, NN_JJ_OUS, NN_JJ_AL \\
\hline NN_JJ_D & NN_RB_D, NN_JJ_D_NN \\
\hline NN_JJ_Y & NN_RB_Y, NN_JJR_Y, NN_JJS_Y, NN_JJ_Y_NN, NN_JJ_Y_NN_ORI \\
\hline NN_J__Y_NN & NN_JJ_Y_NNS \\
\hline NN_JJ_Y_NN_ORI & NN_JJ_Y_NNS_ORI \\
\hline NN_JJ_F & NN_RB_F, NN_JJ_F_NN, NN_JJR_F, NN_JJS_F, NN_JJ_F_NN_ORI \\
\hline NN_J_F_NN & NN_JJ_F_NNS \\
\hline NN_JJ_F_NN_ORI & NN_JJ_F_NNS_ORI \\
\hline NN_JJ_L & NN_RB_L,NN_JJ_L_NN, NN_JJR_L, NN_JJS_L, NN_JJ_L_NN_ORI \\
\hline NN_J_L_NN & NN_JJ_L_NNS \\
\hline NN_JJ_L_NN_ORI & NN_JJ_L_NNS_ORI \\
\hline NN_JJ_AL & NN_RB_AL, NN_JJ_AL_NN \\
\hline NN_JJ_AL_NN & NN_JJ_AL_NNS \\
\hline NN_JJ_OUS & NN_RB_OUS, NN_JJ_OUS_NN \\
\hline NN_JJ_OUS_NN & NN_JJ_OUS_NNS \\
\hline VB & VBP, VBD, VBZ, VBG, VBN \\
\hline VBP & VBP_JJ, VBP_NN_R, VBP_NN_M, VBP_NN_O, VBP_JJ_BLE \\
\hline VBP_JJ & VBP_RB, VBP_JJ_NN, VBP_JJ_NN_ORI \\
\hline VBP_JJ_NN & VBP_JJ_NNS \\
\hline VBP_JJ_NN_ORI & VBP_JJ_NNS_ORI \\
\hline VBP_J_BLE & VBP_RB_BLE VBP_JJ_BLE_NN \\
\hline VBP_JJ_BLE_NN & VBP_JJ_BLE_NNS \\
\hline VBP_NN_R & VBP_NNS_R \\
\hline VBP_NN_M & VBP_NNS_M \\
\hline VBP_NN_O & VBP_NNS_O \\
\hline VBG & VBG_RB, VBG_NNS \\
\hline VBN & VBN_RB, VBN_NN \\
\hline VBN_NN & VBN_NNS \\
\hline JJ & JJR,JJS, RB, JJ_NN, JJ_NN_ORI \\
\hline JJ_NN & JJ_NNS \\
\hline JJ_NN_ORI & JJ_NNS_ORI \\
\hline RB & RBR, RBS \\
\hline CD & CD_JJ, CD_JJ_NN_ORI, CD_NNS \\
\hline CD_JJ & CD_RB, CD_RB_ORI \\
\hline CD_JJ_NN_ORI & CD_JJ_NNS_ORI \\
\hline
\end{tabular}

Table 12: Structure of the EPOS Tree. 